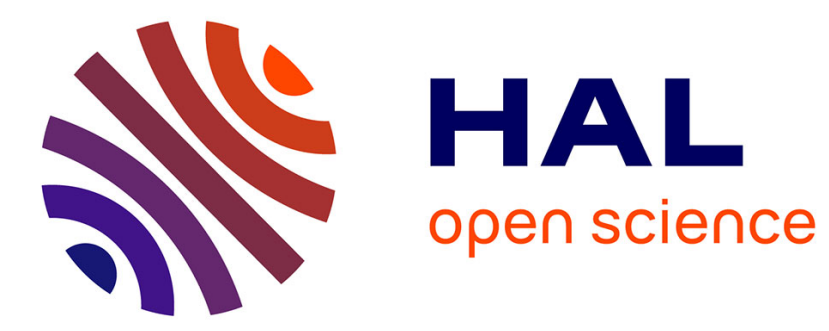

\title{
ELLIOTT-HALBERSTAM CONJECTURE AND VALUES TAKEN BY THE LARGEST PRIME FACTOR OF SHIFTED PRIMES
}

Jie $\mathrm{Wu}$

\section{- To cite this version:}

Jie Wu. ELLIOTT-HALBERSTAM CONJECTURE AND VALUES TAKEN BY THE LARGEST PRIME FACTOR OF SHIFTED PRIMES. Journal of Number Theory, 2020, 206, pp.282-295. 10.1016/j.jnt.2019.06.015 . hal-03216054

\section{HAL Id: hal-03216054 \\ https://hal.science/hal-03216054}

Submitted on 3 May 2021

HAL is a multi-disciplinary open access archive for the deposit and dissemination of scientific research documents, whether they are published or not. The documents may come from teaching and research institutions in France or abroad, or from public or private research centers.
L'archive ouverte pluridisciplinaire HAL, est destinée au dépôt et à la diffusion de documents scientifiques de niveau recherche, publiés ou non, émanant des établissements d'enseignement et de recherche français ou étrangers, des laboratoires publics ou privés. 


\title{
ELLIOTT-HALBERSTAM CONJECTURE AND VALUES TAKEN BY THE LARGEST PRIME FACTOR OF SHIFTED PRIMES
}

\author{
JIE WU
}

\begin{abstract}
Denote by $\mathbb{P}$ the set of all primes and by $P^{+}(n)$ the largest prime factor of integer $n \geqslant 1$ with the convention $P^{+}(1)=1$. For each $\eta>1$, let $c=c(\eta)>1$ be some constant depending on $\eta$ and

$$
\mathcal{P}_{a, c, \eta}:=\left\{p \in \mathbb{P}: p=P^{+}(q-a) \text { for some prime } q \text { with } p^{\eta}<q \leqslant c(\eta) p^{\eta}\right\} .
$$
\end{abstract}

In this paper, under the Elliott-Halberstam conjecture we prove, for $y \rightarrow \infty$,

$$
\pi_{a, c, \eta}(x):=\left|(1, x] \cap \mathcal{P}_{a, c, \eta}\right| \sim \pi(x) \quad \text { or } \quad \pi_{a, c, \eta}(x) \gg_{a, \eta} \pi(x)
$$

according to values of $\eta$. These complement for some results of Banks-Shparlinski [1], of $\mathrm{Wu}[12]$ and of Chen-Wu [2].

\section{INTRODUCTION}

Denote by $\mathbb{P}$ the set of all prime numbers and by $P^{+}(n)$ the largest prime factor of the positive integer $n \geqslant 1$ with the convention $P^{+}(1)=1$. Banks \& Shparlinski [1] proposed to estimate the number of primes $p$ that occur as the largest prime factor of a shifted prime $q-a$ when $q \in \mathbb{P}$ lies in a certain interval determined by $p$. This question has applications in theoretical computer science and has been considered by Vishnoi [10].

Let $\mathbb{Z}^{*}$ be the set of non-zero integers. For $a \in \mathbb{Z}^{*}, c>1$ and $\eta>0$, we put

$$
\mathcal{P}_{a, c, \eta}:=\left\{r \in \mathbb{P}: r=P^{+}(q-a) \text { for some prime } q \text { with } r^{\eta}<q \leqslant c r^{\eta}\right\}
$$

and

$$
\pi_{a, c, \eta}(y):=\left|\left\{r \leqslant y: r \in \mathcal{P}_{a, c, \eta}\right\}\right|, \quad \pi(y):=|\{r \leqslant y: r \in \mathbb{P}\}| .
$$

Banks \& Shparlinski [1, Theorem 1.1] proved that for each $\eta \in\left(\frac{32}{17}, 1+\frac{3}{4} \sqrt{2}\right)$, there exists a constant $c=c(\eta)>1$ such that the asymptotic formula

$$
\pi_{a, c, \eta}(y)=\pi(y)+O_{A, a, c, \eta}\left(\frac{y}{(\log y)^{A}}\right) \quad(y \rightarrow \infty)
$$

holds for every fixed non-zero integer $a \in \mathbb{Z}^{*}$ and any constant $A>1$. Moreover for $2 \leqslant \eta<1+\frac{3}{4} \sqrt{2} \approx 2.0606$, this estimate holds for any constant $c>1$. Very recently, Wu [12] extended Banks-Shparlinski's interval $\left(\frac{32}{17}, 1+\frac{3}{4} \sqrt{2}\right)$ to $\left(\frac{32}{17}, \eta_{0}\right)$, where $\eta_{0} \approx 2.142$ is the unique solution of the equation $\eta-1-4 \eta \log (\eta-1)=0$ in $(1, \infty)$. Banks \& Shparlinski [1, page 144] also remarked that the asymptotic formula (1.1) holds for $\eta \in\left(1, \frac{32}{17}\right]$ if we assume the Elliott-Halberstam conjecture (see $\mathrm{EH}_{\text {prime }}[\varepsilon]$ below). Subsequently, Chen \& Wu

Date: July 2, 2019.

2010 Mathematics Subject Classification. 11N05, 11N25, 11N36.

Key words and phrases. Shifted prime, Friable integer, Sieve. 
[2] further extended the domain of $\eta$ at the price proportion positive instead of density 1. More precisely, they proved that

$$
\pi_{a, c, \eta}(y) \geqslant(\log \sqrt[4]{2}) \frac{\eta-1}{\eta}\left(1-4 \log (\eta-1)-\delta \frac{\log (\eta-1)^{8}}{c-1}\right) \pi(y)
$$

where $\delta=\delta(c, \eta)$ is sufficiently small positive number. Clearly, (1.2) implies

$$
\pi_{a, c, \eta}(y) \gg \pi(y)
$$

provided $\eta<1+\sqrt[4]{\mathrm{e}}$. This is complement for the results of Banks-Shparlinski and of Wu mentioned above. It seems rather natural to pose the following question.

Question 1. Is the asymptotic formula (1.1) true for all $\eta>1$ ?

In this paper, we shall try to answer this question under the well-known Elliott-Halberstam conjecture. Firstly we state two versions of this conjecture for prime numbers.

Conjecture 1 (Elliott-Halberstam). Let $a \in \mathbb{Z}^{*}$ and $\varepsilon \in(0,1)$ be fixed constants.

(i) For any $A>0$, the inequality

$\left(\mathrm{EH}_{\text {prime }}[\varepsilon]\right)$

$$
\sum_{\substack{q \leqslant x^{1-\varepsilon} \\(a, q)=1}}\left|\sum_{\substack{p \leqslant x \\ p \equiv a(\bmod q)}} 1-\frac{\pi(x)}{\varphi(q)}\right| \ll_{A, a, \varepsilon} \frac{x}{(\log x)^{A}}
$$

holds uniformly for all $x \geqslant 3$, where the letter $p$ always denotes prime numbers, $\varphi(q)$ is the Euler function and the implied constant depends on $A, a$ and $\varepsilon$.

(ii) Let $\kappa_{1}(m)$ and $\kappa_{2}(m)$ be the characteristic functions of the odd integers and of even integers, respectively. Then for any $A>0$, we have

$\left(\mathrm{EH}_{\text {prime }}^{*}[\varepsilon]\right)$

$$
\sum_{\substack{q \leqslant x^{1-\varepsilon} \\(a, q)=1}}\left|\sum_{\substack{m p \leqslant x \\ m p \equiv a(\bmod q)}} \kappa_{i}(m)-\frac{1}{\varphi(q)} \sum_{\substack{m p \leqslant x \\(m p, q)=1}} \kappa_{i}(m)\right| \ll_{A, a, \varepsilon} \frac{x}{(\log x)^{A}}
$$

uniformly for all $x \geqslant 3$, where the letter $p$ always denotes prime numbers and the implied constant depends on $A$ and $a$.

Remark 1. According to the classical Bombieri-Vinogradov theorem and Proposition 2.2 of $\mathrm{Wu}[12]$, the Elliott-Halberstam conjectures $\mathrm{EH}_{\text {prime }}[\varepsilon]$ and $\mathrm{EH}_{\text {prime }}^{*}[\varepsilon]$ hold for all $\varepsilon \in\left(\frac{1}{2}, 1\right)$.

Secondly we also need a version of this conjecture for friable numbers.

Conjecture 2 (Elliott-Halberstam). Let $a \in \mathbb{Z}^{*}$ and $\varepsilon \in(0,1)$ be fixed constants. For any $A>0$, we have

$$
\left(\mathrm{EH}_{\text {friable }}[\varepsilon]\right) \quad \sum_{\substack{q \leqslant x^{1-\varepsilon} \\(a, q)=1}}\left|\sum_{\substack{n \leqslant x \\ n \equiv a(\bmod q), P^{+}(n) \leqslant y}} 1-\frac{1}{\varphi(q)} \sum_{\substack{n \leqslant x \\(n, q)=1, P^{+}(n) \leqslant y}} 1\right| \ll_{A, a, \varepsilon} \frac{x}{(\log x)^{A}}
$$

uniformly in $x \geqslant y \geqslant 2$.

Remark 2. According to Wolke's work [11] (see also [4, Theorem 6]), $\mathrm{EH}_{\text {friable }}[\varepsilon]$ holds unconditionally for all $\varepsilon \in\left(\frac{1}{2}, 1\right)$. 
Our results are as follows.

Theorem 1. Let $a \in \mathbb{Z}^{*}$ and $c>1$ be fixed constants.

(i) Let $\eta \in\left(1, \frac{32}{17}\right]$ and assume the Elliott-Halberstam conjecture $\mathrm{EH}_{\text {prime }}[\varepsilon]$ with $\varepsilon=1-$ $1 / \eta>0$. Then for any $A>1$ we have

$$
\pi_{a, c, \eta}(y)=\pi(y)+O_{A, a, c, \eta}\left(\frac{y}{(\log y)^{A}}\right)
$$

as $y \rightarrow \infty$.

(ii) Let $\eta_{1} \approx 2.3303$ be the unique positive zero of the equation $\eta-1-2 \eta \log (\eta-1)=0$ in $(1, \infty)$. For each $\eta \in\left[\eta_{0}, \eta_{1}\right)$, there is a sufficiently small positive number $\varepsilon=\varepsilon(\eta)$ such that assuming the Elliott-Halberstam conjecture $\mathrm{EH}_{\text {prime }}[\varepsilon]$, for any $A>1$ we have

$$
\pi_{a, c, \eta}(y)=\pi(y)+O_{A, a, c, \eta}\left(\frac{y}{(\log y)^{A}}\right)
$$

as $y \rightarrow \infty$.

Theorem 2. Let $a \in \mathbb{Z}^{*}$ and $c>1$ be fixed constants. For every $\eta \in[2, \infty)$, there is a sufficiently small positive number $\varepsilon=\varepsilon(\eta)$ such that under the Elliott-Halberstam conjecture $\mathrm{EH}_{\text {friable }}[\varepsilon]$, we have

$$
\pi_{a, c, \eta}(y) \geqslant(\log \sqrt[4]{2}) \frac{\eta-1}{\eta} \pi(y)\left\{1+O_{a, c, \eta}\left(\frac{1}{\sqrt[3]{\log y}}+\varepsilon\right)\right\}
$$

as $y \rightarrow \infty$.

Remark 3. (a) For comparaison, we have

$$
1+\frac{3}{4} \sqrt{2} \approx 2.060, \quad \eta_{0} \approx 2.142, \quad \eta_{1} \approx 2.330, \quad 1+\sqrt[4]{\mathrm{e}} \approx 2.284, \quad 1+\sqrt{\mathrm{e}} \approx 2.648 .
$$

(b) The first assertion of Theorem 1 is due to Banks \& Shparlinski [1, page 144]. Here we give a proof for convenience of the reader. By combining Theorem 1 with results of [12], we see that the asymptotic formula (1.1) holds for $1<\eta<\eta_{1}$ under the Elliott-Halberstam conjecture.

(c) Theorem 2 improves signaficantively Chen-Wu's result (1.2) in two aspects : result and method. Firstly the proportion in (1.5) increases from $\frac{\log 2}{8}$ to $\frac{\log 2}{4}$ when $\eta$ runs over

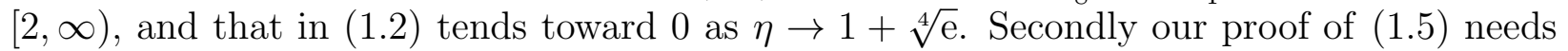
information of prime numbers in "arithmetical progressions" $\{a+m q\}_{m}$ friable with friable indice. For $(a, q)=1$ and $x \geqslant y \geqslant 2$, define the counting function

$$
\pi(x, y ; q, a):=\sum_{\substack{p \leqslant x \\ p \equiv a(\bmod q) \\ P^{+}((p-a) / q) \leqslant y}} 1 .
$$

A systematic study on the asymptotical behaviour has been done by Liu, Wu \& Xi [8], recently. We need a theorem of Bombieri-Vinogradov type and an inequality of BrunTitichmarsh for this new counting function (see Lemmas 2.2-2.3 below). 


\section{SOME PRELIMINARY LEMMAS}

In this section, we present three lemmas, which will be useful later.

\subsection{The Rosser-Iwaniec linear sieve.}

The first lemma is due to Iwaniec $[6,7]$.

Lemma 2.1. Let $D \geqslant 2$ and let $\mu(n)$ be the Möbius function. Then there are two sequences $\left\{\lambda_{d}^{ \pm}\right\}_{d \geqslant 1}$, vanishing for $d>D$ or $\mu(d)=0$, satisfying $\left|\lambda_{d}^{ \pm}\right| \leqslant 1$, such that

$$
\sum_{d \mid n} \lambda_{d}^{-} \leqslant \sum_{d \mid n} \mu(d) \leqslant \sum_{d \mid n} \lambda_{d}^{+} \quad(n \geqslant 1)
$$

and

$$
\begin{gathered}
\sum_{d \mid P_{\mathcal{P}}(z)} \lambda_{d}^{+} \frac{w(d)}{d} \leqslant \prod_{\substack{p \leqslant z \\
p \in \mathcal{P}}}\left(1-\frac{w(p)}{p}\right)\left\{F(s)+O\left(\frac{\mathrm{e}^{\sqrt{L}-s}}{\sqrt[3]{\log D}}\right)\right\} \\
\sum_{d \mid P_{\mathcal{P}}(z)} \lambda_{d}^{-} \frac{w(d)}{d} \geqslant \prod_{\substack{p \leqslant z \\
p \in \mathcal{P}}}\left(1-\frac{w(p)}{p}\right)\left\{f(s)+O\left(\frac{\mathrm{e}^{\sqrt{L}-s}}{\sqrt[3]{\log D}}\right)\right\}
\end{gathered}
$$

for any $z \in[2, D], s=(\log D) / \log z$, set of prime numbers $\mathcal{P}$ and multiplicative function $w$ satisfying

$$
\begin{gathered}
0<w(p)<p \quad(p \in \mathcal{P}), \\
\prod_{u<p \leqslant v, p \in \mathcal{P}}\left(1-\frac{w(p)}{p}\right)^{-1} \leqslant \frac{\log v}{\log u}\left(1+\frac{L}{\log u}\right) \quad(2 \leqslant u \leqslant v),
\end{gathered}
$$

where $P_{\mathcal{P}}(z):=\prod_{p \leqslant z, p \in \mathcal{P}} p$ and the implied $O$-constants are absolute. Here $F, f$ are defined by the continuous solutions to the system

$$
\begin{cases}s F(s)=2 \mathrm{e}^{\gamma} & (1 \leqslant s \leqslant 2) \\ s f(s)=0 & (0<s \leqslant 2) \\ (s F(s))^{\prime}=f(s-1) & (s>2) \\ (s f(s))^{\prime}=F(s-1) & (s>2)\end{cases}
$$

where $\gamma$ is the Euler constant.

\subsection{Bombieri-Vinogradov theorem of for $\pi(x, y ; q, a)$.}

The second lemma is a theorem of Bombieri-Vinogradov type for the counting function $\pi(x, y ; q, a)$ defined as in (1.6) (see [8, Theorem 2]).

Lemma 2.2. Let $a \in \mathbb{Z}^{*}, A>0$ and $\kappa$ a non-negative arithmetic function. Assuming the Elliott-Halberstam conjecture $\mathrm{EH}_{\text {prime }}[\varepsilon]$, the following estimate

$$
\sum_{\substack{q \leqslant Q \\(q, a)=1}} \kappa(q)\left|\pi(x, y ; q, a)-\frac{\pi(x)}{\varphi(q)} \rho\left(\frac{\log (x / q)}{\log y}\right)\right| \ll_{a, A} \frac{x}{(\log x)^{A}} \sqrt{\sum_{q \leqslant x} \frac{\kappa(q)^{2}}{q}}+\pi(x) \varepsilon u \sum_{q \leqslant Q} \frac{\kappa(q)}{\varphi(q)}
$$

holds uniformly in $x \geqslant 2$, $\exp \left\{(\log x)^{2 / 5+\varepsilon}\right\} \leqslant y \leqslant x$ and $Q \leqslant \min \{y, \sqrt{x}\}$, where $\rho(u)$ is the Dickman function. 


\subsection{Brun-Titichmarsh inequality for $\pi(x, y ; q, a)$.}

The lemma below is a variant of [8, Theorem 1].

Lemma 2.3. Let $a \in \mathbb{Z}^{*}$ and $c>1$ be fixed constants. For any $\varepsilon>0$, we have

$$
\begin{gathered}
\pi(c x, y ; q, a)-\pi(x, y ; q, a) \leqslant \frac{4(c-1) x}{\varphi(q) \log (x / q)} \rho\left(\frac{\log (x / q)}{\log y}\right)\left\{1+O_{a, c, \varepsilon}\left(\frac{1}{\sqrt[3]{\log x}}\right)\right\} \\
+O_{A, a, c}\left(\frac{x}{q(\log x)^{A}}\right)
\end{gathered}
$$

uniformly in $\left.\exp \{\log \log x)^{5 / 3+\varepsilon}\right\} \leqslant y \leqslant x$ and $1 \leqslant q \leqslant \min \{y, \sqrt{x}\}$ with $(a, q)=1$.

Proof. Denote by $\mathcal{S}$ the quantity on the left-hand side of (2.6). Without loss of generality, we can assume $q$ is even and $a$ is odd. Put $P_{2 a}(z):=\prod_{p \leqslant z, p \nmid 2 a} p$. By the Möbius inversion, we can write

$$
\begin{aligned}
\mathcal{S} & =\sum_{\substack{x<a+m q \leqslant c x \\
(m q, a)=\left(a+m q, P_{2}(\sqrt{c x})\right)=1 \\
P^{+}(m) \leqslant y}} 1+O\left(x^{\varepsilon}\right) \\
& =\sum_{\substack{x<a+m q \leqslant c x \\
(a, m q)=1, P^{+}(m) \leqslant y}} \sum_{\substack{\left(a+m q, P_{2 a}(\sqrt{c x})\right)\\
}} \mu(d)+O\left(x^{\varepsilon}\right),
\end{aligned}
$$

Using Lemma 2.1 and switching summations, it follows that

$$
\begin{aligned}
\mathcal{S} & \leqslant \sum_{\substack{x<a+m q \leqslant c x \\
(a, m q)=1, P^{+}(m) \leqslant y}} \sum_{\substack{d \mid\left(a+m q, P_{2 a}(\sqrt{c x})\right) \\
d \leqslant D}} \lambda_{d}^{+}+O\left(x^{\varepsilon}\right) \\
& \lambda_{d \mid P_{2 a q}(\sqrt{c x})}^{+} \sum_{\substack{(x-a) / q<m \leqslant(c x-a) / q \\
m \equiv-a \bar{q}(\bmod d) \\
(a, m)=1, P^{+}(m) \leqslant y}} 1+O\left(x^{\varepsilon}\right),
\end{aligned}
$$

where $\left\{\lambda_{d}^{+}\right\}_{d \geqslant 1}$ is an upper bound sieve of level $D$ as in Lemma 2.1 and $\bar{q}$ is the inverse of $q$ modulo $d$ (i.e. $q \bar{q} \equiv 1(\bmod d)$ ). To apply the Elliott-Halberstam conjecture $\mathrm{EH}_{\text {friable }}[\varepsilon]$, we would like to remove the restriction $(a, m)=1$ by Möbius inversion, so that

$$
\begin{aligned}
\mathcal{S} & \leqslant \sum_{\ell \mid a} \mu(\ell) \sum_{\substack{d \leqslant D \\
d \mid P_{2 a q}(\sqrt{c x})}} \lambda_{d}^{+} \sum_{\substack{(x-a) / q<m \leqslant(c x-a) / q \\
m \equiv-a \bar{q}(\bmod d) \\
\ell \mid m, P^{+}(m) \leqslant y}} 1+O\left(x^{\varepsilon}\right) \\
& =\sum_{\substack{\ell \mid a \\
P^{+}(\ell) \leqslant y}} \mu(\ell) \sum_{\substack{d \leqslant D \\
d \mid P_{2 a q}(\sqrt{c x})}} \lambda_{d}^{+} \sum_{\substack{(x-a) / \ell q<m \leqslant(c x-a) / \ell q \\
m \equiv-a \overline{\ell q}(\bmod d) \\
P^{+}(m) \leqslant y}} 1+O\left(x^{\varepsilon}\right) .
\end{aligned}
$$

We are now in a good position to employ the Elliott-Halberstam conjecture $\mathrm{EH}_{\text {friable }}[\varepsilon]$ with $D=(x / q)^{1-\varepsilon}$, getting

$$
\mathcal{S} \leqslant \mathcal{S}^{+}+O\left(\frac{x}{q(\log x)^{A}}\right)
$$


where

$$
\mathcal{S}^{+}:=\sum_{\ell \mid a} \mu(\ell) \sum_{\substack{d \leqslant D \\ d \mid P_{2 a q}(\sqrt{c x})}} \frac{\lambda_{d}^{+}}{\varphi(d)} \sum_{\substack{x / \ell q<m \leqslant c x / \ell q \\(d, m)=1, P^{+}(m) \leqslant y}} 1
$$

and we have used the trivial bound

$$
\sum_{\ell \mid a} \sum_{\substack{d \leqslant D \\ d \mid P_{2 a q}(\sqrt{c x})}} \frac{\left|\lambda_{d}^{+}\right|}{\varphi(d)} \sum_{\substack{c x / \ell q<m \leqslant(c x-a) / \ell q \\(d, m)=1, P^{+}(m) \leqslant y}} 1 \ll_{a} \log x
$$

Here we removed the restriction that $P^{+}(\ell) \leqslant y$ since we henceforth assume $y>|a|$.

Switching summations, it follows that

$$
\begin{aligned}
\mathcal{S}^{+} & =\sum_{\ell \mid a} \mu(\ell) \sum_{\substack{x / \ell q<m \leqslant c x / \ell q \\
P^{+}(m) \leqslant y}} \sum_{\substack{d \leqslant D \\
d \mid P_{2 a m q}(\sqrt{c x})}} \frac{\lambda_{d}^{+}}{\varphi(d)} \\
& =\sum_{\substack{x / q<m \leqslant c x / q \\
(a, m)=1, P^{+}(m) \leqslant y}} \sum_{\substack{d \leqslant D \\
d \mid P_{2 a m q}(\sqrt{c x})}} \frac{\lambda_{d}^{+}}{\varphi(d)} .
\end{aligned}
$$

From Lemma 2.1, we deduce

$$
\begin{aligned}
\mathcal{S}^{+} & \leqslant \frac{\log \sqrt{x}}{\log (x / q)}\left\{2 \mathrm{e}^{\gamma}+O\left(\frac{1}{\sqrt[3]{\log x}}\right)\right\} \sum_{\substack{x / q<m \leqslant c x / q \\
(a, m)=1, P^{+}(m) \leqslant y}} \prod_{\substack{p<2 a m q \\
p \nmid 2 a m q}}\left(1-\frac{1}{p-1}\right) \\
& \leqslant \frac{\log \sqrt{x}}{\log (x / q)}\left\{2 \mathrm{e}^{\gamma}+O\left(\frac{1}{\sqrt[3]{\log x}}\right)\right\} \prod_{\substack{p<\sqrt{x} \\
p \nmid 2 a q}}\left(1-\frac{1}{p-1}\right) \sum_{\substack{x / q<m \leqslant c x / q \\
(a, m)=1, P^{+}(m) \leqslant y}} H(m),
\end{aligned}
$$

where $H(m)$ is the multiplicative function, defined by

$$
H\left(p^{\nu}\right)= \begin{cases}1 & \text { if } p \mid 2 q \text { or } p>x^{1 / 2} \\ \frac{p-1}{p-2} & \text { if } p \nmid 2 q \text { and } p \leqslant x^{1 / 2}\end{cases}
$$

for all $\nu \geqslant 1$. According to $[8,(3.5)]$, we have

$$
\sum_{\substack{m \in S(x / q, y) \\(a, m)=1}} H(m)=\Psi\left(\frac{x}{q}, y\right) \frac{\varphi(a)}{a} \prod_{\substack{p<\sqrt{x} \\ p \nmid 2 a q}}\left(1+\frac{1}{p(p-2)}\right)\left\{1+O\left(\frac{(\log \log x)^{2}}{\log y}\right)\right\}
$$

where $S(x, y):=\left\{n \leqslant x: P^{+}(n) \leqslant y\right\}$ and $\Psi(x, y):=|S(x, y)|$. Combining this with (2.8), we find that

$$
\mathcal{S}^{+} \leqslant \frac{\log x}{\log (x / q)}\left\{2 \mathrm{e}^{\gamma}+O\left(\frac{1}{\sqrt[3]{\log x}}\right)\right\} \frac{\varphi(a)}{a}\left\{\Psi\left(\frac{c x}{q}, y\right)-\Psi\left(\frac{x}{q}, y\right)\right\} \prod_{\substack{p \leqslant \sqrt{x} \\ p \nmid a q}}\left(1-\frac{1}{p}\right) .
$$


By the Mertens formula, it follows that

$$
\prod_{\substack{p \leqslant x^{1 / 2} \\ p \nmid a q}}\left(1-\frac{1}{p}\right)=\frac{a q}{\varphi(a) \varphi(q)} \cdot \frac{2 \mathrm{e}^{-\gamma}}{\log x}\left\{1+O\left(\frac{1}{\log x}\right)\right\} .
$$

On the other hand, according to [5, Theorem 1], we have

$$
\Psi(x, y)=x \rho\left(\frac{\log x}{\log y}\right)\left\{1+O\left(\frac{\log ((\log x) / \log y+1)}{\log y}\right)\right\}
$$

uniformly for $x \geqslant 3$ and $\exp \left\{(\log \log x)^{5 / 3+\varepsilon}\right\} \leqslant y \leqslant x$. Combining these with (2.9) and (2.7), we can get the required inequality (2.6).

\section{Proof of TheOREM 2}

For each prime $r \in\left(\frac{1}{2} y, y\right]$, consider

$$
Q_{r}(y):=\sum_{\substack{x<q \leqslant c x \\ P^{+}(q-a)=r}} 1 .
$$

Noticing that

$$
P^{+}(q-a)=r \Leftrightarrow q \equiv a(\bmod r) \text { and } P^{+}(q-a) \leqslant r
$$

we can write

$$
\begin{aligned}
\sum_{y<r \leqslant 2 y} \mathcal{Q}_{r}(y) & \geqslant \sum_{y<r \leqslant 2 y} \sum_{\substack{x<q \leqslant c x \\
q \equiv a(\bmod r), P^{+}(q-a) \leqslant y}} 1 \\
& =\sum_{y<r \leqslant 2 y}(\pi(c x, y ; r, a)-\pi(x, y ; r, a)) \\
& =\mathcal{M}+\mathcal{E},
\end{aligned}
$$

where

$$
\begin{aligned}
\mathcal{M} & :=\sum_{y<r \leqslant 2 y}\left(\frac{\pi(c x)}{\varphi(r)} \rho\left(\frac{\log (c x / r)}{\log y}\right)-\frac{\pi(x)}{\varphi(r)} \rho\left(\frac{\log (x / r)}{\log y}\right)\right), \\
\mathcal{E} & :=\sum_{y<r \leqslant 2 y}(\mathcal{E}(c x, y ; r, a)-\mathcal{E}(x, y ; r, a)),
\end{aligned}
$$

and

$$
\mathcal{E}(x, y ; r, a):=\pi(x, y ; r, a)-\frac{\pi(x)}{\varphi(r)} \rho\left(\frac{\log (x / r)}{\log y}\right) .
$$

Since $\eta \geqslant 2$, we have $y=x^{1 / \eta} \leqslant x^{1 / 2}$ and $Q=\min (y, \sqrt{x})=y$. Using Lemma 2.2 with the characteristic function of prime numbers in $(y, 2 y]$ in place of $\kappa(q)$, we easily derive, under the conjecture of Elliott-Halberstam $\mathrm{EH}_{\text {prime }}[\varepsilon]$, that

$$
\begin{aligned}
|\mathcal{E}| & \leqslant \sum_{y<r \leqslant 2 y}(|\mathcal{E}(c x, y ; r, a)|+|\mathcal{E}(x, y ; r, a)|) \\
& \ll_{a} \frac{x}{(\log x)^{3}}+\varepsilon \eta \frac{\pi(x)}{\log y} \ll_{a, \eta} \varepsilon \frac{\pi(x)}{\log y}
\end{aligned}
$$


for all $x \geqslant x_{0}(\varepsilon)$, where we have used the following bound

$$
\sum_{y<r \leqslant 2 y} \frac{1}{\varphi(r)}=\sum_{y<r \leqslant 2 y} \frac{1}{r}\left\{1+O\left(\frac{1}{y}\right)\right\}=\frac{\log 2}{\log y}\left\{1+O\left(\frac{1}{\log y}\right)\right\}
$$

and the implied constant depends on $a, \eta$ at most.

According to [9, Corollary III.5.8.3], we have $\left|\rho^{\prime}(u)\right| \ll \rho(u) \log u(u>1)$. Thus for all $r \in(y, 2 y]$, we have

$$
\rho\left(\frac{\log (x / r)}{\log y}\right)=\rho(\eta-1)\left\{1+O_{\eta}\left(\frac{1}{\log y}\right)\right\} .
$$

From this and (3.4), we derive

$$
\mathcal{M}=(\log 2)(c-1) \rho(\eta-1) \frac{\pi(x)}{\log y}\left\{1+O_{\eta}\left(\frac{1}{\log y}\right)\right\} .
$$

Inserting (3.5) and (3.3) into (3.2), it follows that

$$
\sum_{y<r \leqslant 2 y} Q_{r}(y) \geqslant(\log 2)(c-1) \rho(\eta-1) \frac{\pi(x)}{\log y}\left\{1+O_{a, c, \eta}\left(\frac{1}{\log y}+\varepsilon\right)\right\} .
$$

On the other hand, the Brun-Titchmarsh inequality (2.6) give us

$$
\begin{aligned}
\mathcal{Q}_{r}(y) & \leqslant \frac{4(c-1) x}{\varphi(r) \log (x / r)} \rho(\eta-1)\left\{1+O_{a, \eta}\left(\frac{1}{\sqrt[3]{\log x}}\right)\right\} \\
& \leqslant 4(c-1) \frac{\rho(\eta-1)}{\eta-1} \cdot \frac{x}{y \log y}\left\{1+O_{a, \eta}\left(\frac{1}{\sqrt[3]{\log x}}\right)\right\}
\end{aligned}
$$

for all primes $r \in(y, 2 y]$. This implies that

$$
\sum_{y<r \leqslant 2 y} Q_{r}(y) \leqslant \rho(\eta-1) \frac{4(c-1)}{(\eta-1)} \cdot \frac{x}{y \log y}\left\{1+O_{a, \eta}\left(\frac{1}{\sqrt[3]{\log x}}\right)\right\} \sum_{\substack{y<r \leqslant 2 y \\ Q_{r}(y) \neq 0}} 1 .
$$

Combining (3.6) and (3.7), it follows that

$$
\sum_{\substack{y<r \leqslant 2 y \\ Q_{r}(y) \neq 0}} 1 \geqslant(\log \sqrt[4]{2}) \frac{\eta-1}{\eta} \pi(y)\left\{1+O_{a, c, \eta}\left(\frac{1}{\sqrt[3]{\log x}}+\varepsilon\right)\right\} .
$$

This completes the proof of Theorem 2 .

\section{Proof of TheOREM 1}

As in [12], the letters $p, q, r$ and $\ell$ are always used to denote prime numbers, and $d, m$, and $n$ always denote positive integers. In what follows, let $a \in \mathbb{Z}^{*}$ and $\eta \in\left(1, \frac{32}{17}\right] \cup\left[\eta_{0}, \eta_{1}\right)$. Let $\delta$ be a sufficiently small positive constant and let $c>1$ be a parameter to be chosen later. Let $x_{0}(A, a, c, \eta, \delta)$ be a large constant depending on $A, a, c, \eta, \delta$ at most. For $x \geqslant x_{0}(A, a, c, \eta, \delta)$ and $r \in\left(\frac{1}{2} y, y\right]$, put $x:=r^{\eta}$. As usual, for $(a, d)=1$ define

$$
\pi(x ; d, a):=\sum_{\substack{p \leqslant x \\ p \equiv a(\bmod d)}} 1 .
$$


4.1. The case of $\eta \in\left(1, \frac{32}{17}\right]$.

For $\eta>1, c>1, y \geqslant 3$ and $x=y^{\eta}$, put

$$
\begin{aligned}
& \mathcal{R}_{\mathrm{b}}^{\prime}(y):=\left\{y<r \leqslant 2 y:\left|\pi(x ; r, a)-\frac{\pi(x)}{\varphi(r)}\right| \geqslant \delta \frac{\pi(x)}{\varphi(r)}\right\}, \\
& \mathcal{R}_{\mathrm{b}}^{\prime \prime}(y):=\left\{y<r \leqslant 2 y:\left|\pi(c x ; r, a)-\frac{\pi(c x)}{\varphi(r)}\right| \geqslant \delta \frac{\pi(c x)}{\varphi(r)}\right\} .
\end{aligned}
$$

Noticing that $y=x^{1 / \eta}=x^{1-(1-1 / \eta)}$, the Elliott-Halberstam conjecture $\mathrm{EH}_{\text {prime }}[\varepsilon]$ with $\varepsilon=$ $1-1 / \eta$ allows us to deduce that

$$
\begin{aligned}
\delta \frac{\pi(x)}{y}\left|\mathcal{R}_{\mathrm{b}}^{\prime}(y)\right| & \leqslant \sum_{y<r \leqslant 2 y}\left|\pi(x ; r, a)-\frac{\pi(x)}{\varphi(r)}\right| \\
& \ll_{A, a, \delta, \eta} \frac{x}{(\log x)^{A+1}},
\end{aligned}
$$

which gives immediately

$$
\left|\mathcal{R}_{\mathrm{b}}^{\prime}(y)\right| \ll_{A, a, \delta, \eta} \frac{y}{(\log y)^{A}} .
$$

Similarly

$$
\left|\mathcal{R}_{\mathrm{b}}^{\prime \prime}(y)\right| \ll_{A, a, c, \delta, \eta} \frac{y}{(\log y)^{A}}
$$

Define

$$
\begin{aligned}
& \mathcal{R}_{\mathrm{g}}^{\prime}(y):=\left\{y<r \leqslant 2 y: \pi(x ; r, a) \leqslant(1+\delta) \frac{\pi(x)}{\varphi(r)}\right\} \\
& \mathcal{R}_{\mathrm{g}}^{\prime \prime}(y):=\left\{y<r \leqslant 2 y: \pi(c x ; r, a) \geqslant(1-\delta) \frac{\pi(c x)}{\varphi(r)}\right\},
\end{aligned}
$$

and

Clearly

$$
\mathcal{R}_{\mathrm{g}}(y):=\mathcal{R}_{\mathrm{g}}^{\prime}(y) \cap \mathcal{R}_{\mathrm{g}}^{\prime \prime}(y)
$$

$$
\mathcal{R}_{\mathrm{g}}(y) \subset \mathbb{P} \cap(y, 2 y] \subset \mathcal{R}_{\mathrm{b}}^{\prime}(x) \cup \mathcal{R}_{\mathrm{b}}^{\prime \prime}(x) \cup \mathcal{R}_{\mathrm{g}}(y) .
$$

Thus the estimations (4.1) and (4.2) imply that

$$
\left|\mathcal{R}_{\mathrm{g}}(y)\right|=\pi(2 y)-\pi(y)+O_{A, a, c, \delta, \eta}\left(\frac{y}{(\log y)^{A}}\right) \quad(y \geqslant 2)
$$

Let $r \in \mathcal{R}_{\mathrm{g}}(y)$ and let $\mathcal{Q}_{r}(y)$ be defined as in (3.1). When $\eta \in\left(1, \frac{32}{17}\right]$, we have $r>y=$ $x^{1 / \eta} \geqslant x^{17 / 32}>(c x)^{1 / 2}$. Thus the definition of $\mathcal{R}_{\mathrm{g}}(y)$ allows us to write

$$
\mathcal{Q}_{r}(y)=\pi(c x ; r, a)-\pi(x ; r, a) \geqslant(c-1-3 \delta) \frac{\pi(x)}{\varphi(r)}>0
$$

where we have used the inequality $\pi(c x) \geqslant(c-\delta) \pi(x)$ for $x \geqslant x_{0}(a, c, \delta)$. By the definition of $\mathcal{P}_{a, c, \eta}$ and (4.4), it is easy to see that $\mathcal{R}_{\mathrm{g}}(y) \subset \mathcal{P}_{a, c, \eta} \cap[y, 2 y]$. In view of (4.3), we find that

$$
\pi_{a, c, \eta}(2 y)-\pi_{a, c, \eta}(y)=\pi(2 y)-\pi(y)+O_{A, a, c, \eta}\left(\frac{y}{(\log y)^{A}}\right) .
$$

This implies the first assertion of Theorem 1, thanks to standard dyadic split. 
4.2. The case of $\eta \in\left[\eta_{0}, \eta_{1}\right)$.

In this case, for every prime $r \in \mathcal{R}_{\mathrm{g}}(y)$, we can write

$$
\begin{aligned}
\mathcal{Q}_{r}(y) & =\pi(c x ; r, a)-\pi(x ; r, a)-\mathscr{Q}_{r}(y) \\
& \geqslant(c-1-3 \delta) \frac{\pi(x)}{\varphi(r)}-\mathscr{Q}_{r}(y) .
\end{aligned}
$$

for $x \geqslant x_{0}(a, c, \delta)$, where

$$
\mathscr{Q}_{r}(y):=\sum_{\substack{x<q \leqslant c x \\ q \equiv a(\bmod r), P(q-a)>r}} 1 .
$$

Similar to $\left[12\right.$, Proposition 2.1] ${ }^{*}$, we can prove

$$
\mathscr{Q}_{r}(y) \leqslant(c-1+2 \delta) \frac{2 \eta \log (\eta-1)}{\eta-1} \cdot \frac{\pi(y)}{\varphi(r)}\left\{1+O_{a, c, \delta, \eta, \varepsilon}\left(\frac{1}{\sqrt[3]{\log r}}\right)\right\}
$$

for $y \geqslant 3, r \in(y, 2 y]$ and $\eta \geqslant 2$.

Inserting (4.7) into (4.4) and taking $c=1+2 \sqrt{\delta}$, we can find that

$$
\begin{aligned}
\mathcal{Q}_{r}(y) & \geqslant 2 \frac{\sqrt{\delta}-\delta}{\eta-1}\left(\eta-1-2 \eta \log (\eta-1) \cdot \frac{1+\sqrt{\delta}}{1-\sqrt{\delta}}\right) \frac{\pi(y)}{\varphi(r)} \\
& =\{G(\eta)+O(\sqrt{\delta})\} 2 \sqrt{\delta} \frac{1-\sqrt{\delta}}{\eta-1} \cdot \frac{\pi(y)}{\varphi(r)}
\end{aligned}
$$

where

$$
G(\eta):=\eta-1-2 \eta \log (\eta-1)
$$

It is easy to see that $G(\eta)$ is decreasing on $[2, \infty)$ and $G(2)=1$. Therefore there is a unique real number $\eta_{1} \in(2, \infty)$ such that $G\left(\eta_{1}\right)=0$ and for $\eta \in\left[2, \eta_{1}\right)$ we have the inequality

$$
\mathcal{Q}_{r}(y) \gg_{A, a, \delta, \eta} \frac{\pi(y)}{\varphi(r)}
$$

for $y \geqslant y_{0}(A, a, \delta, \eta)$. As before, (4.9) allows us to deduce that $\mathcal{R}_{\mathrm{g}}(y) \subseteq \mathcal{P}_{a, c, \eta} \cap(y, 2 y]$. Combining this with (4.3) leads to

$$
\pi_{a, c, \eta}(2 y)-\pi_{a, c, \eta}(y)=\pi(2 y)-\pi(y)+O_{A, a, c, \delta, \eta}\left(\frac{y}{(\log y)^{A}}\right) .
$$

This implies the required asymptotic formula (1.4).

Acknowledgements. This work is supported in part by Scientific Research Innovation Team Project Affiliated to Yangtze Normal University (No. 2016XJTD01).

${ }^{*}$ The proof is identical and the only difference is that we can take $z=(y / q)^{(1-\varepsilon) / 2}$ and $D=z^{2}$ thanks to the Elliott-Halberstam conjecture $\mathrm{EH}_{\text {prime }}^{*}[\varepsilon]$, instead of $z=(y / q)^{1 / 4} /(\log y)^{B}$ and $D=z^{2}$ 


\section{REFERENCES}

[1] W. Banks \& Igor E. Shparlinski, On values taken by the largest prime factor of shifted primes, J. Aust. Math. Soc. 82 (2007), 133-147.

[2] B. Chen \& J. Wu, On values taken by the largest prime factor of shifted primes (II), International J. Number Theory, 15 (2019), no. 5, 935-944.

[3] B. Feng \& J. Wu, On the density of shifted primes with large prime factors, Science China Mathematics, 61 (2018), no. 1, 83-94.

[4] É. Fouvry \& G. Tenenbaum, Entiers sans grand facteur premier en progressions arithmétiques, Proc. London Math. Soc. 63 (1991) 449-494.

[5] A. Hildebrand, On the number of positive integers $\leqslant x$ and free of prime factors $>y$, J. Number Theory, 22 (1986), 289-307.

[6] H. Iwaniec, Rosser's sieve, Acta Arith., 36 (1980), 171-202.

[7] H. Iwaniec, A new form of the error term in the linear sieve, Acta Arith., 37 (1980), 307-320.

[8] Jianya Liu, Jie Wu \& Ping Xi, Primes in arithmetic progressions with friable indices, Science China Mathematics, to appear.

[9] G. Tenenbaum, Introduction to analytic and probabilistic number theory, Translated from the second French edition (1995) by C. B. Thomas, Cambridge Studies in Advanced Mathematics 46, Cambridge University Press, Cambridge, 1995. xvi+448 pp.

[10] N. K. Vishnoi, Theoretical aspects of randomization in computation, Ph. D. Thesis, Georgia Inst. of Tchnogy, 2004. (http://smartech.gatech.edu:8282/dspace/handle/1853/5049)

[11] D. Wolke, Über die mittlere Verteilung der Werte zahlentheoretischer Funktionen auf Restklassen. II, Math. Ann. 204 (1973), 145-153.

[12] J. Wu, On values taken by the largest prime factor of shifted primes, J. Aust. Math. Soc., to appear. doi:10.1017/S144678871800023X.

School of Mathematics and Statistics, Yangtze Normal University, Fuling, Chongqing 408100, China

Current address: CNRS LAMA 8050, Laboratoire d'Analyse et de Mathématiques Appliquées, Université Paris-Est Créteil, 94010 Créteil Cedex, France

E-mail address: jie.wu@math.cnrs.fr 